\title{
Grandeurs de protection en dosimétrie externe*
}

\author{
D. PAUL**
}

(Manuscrit reçu le 14 janvier 2000, révisé le 5 mai 2000, accepté le 10 mai 2000)

\begin{abstract}
RÉSUMÉ Cet article résume les principales recommandations tirées des dernières publications de l'ICRP et de l'ICRU sur les grandeurs de protection et les grandeurs opérationnelles. L'objectif est de focaliser l'attention sur les relations qui existent entre ces grandeurs à des fins d'étalonnage en radioprotection et d'analyser le comportement de l'instrumentation utilisée pour la mesure des équivalents de dose dus aux sources externes de photons et d'électrons
\end{abstract}

ABSTRACT Protection quantities in external dosimetry.

This paper summarises the main recommendations from the last publications of ICRP and ICRU on protection and operational quantities. The objective is to focus on the relationship of these quantities for radiological protection monitoring purposes, and to analyse the behaviour of the instrumentation used for the measurement of dose equivalents coming from external photon and electron radiations.

\section{Introduction}

1991, l'ICRP (International Commission on Radiological Protection) introduit avec sa publication numéro 60 un nouveau système de protection radiologique, source d'inspiration de la Directive européenne 96/29/Euratom du 13 mai 1996 «fixant les normes de base relatives à la protection sanitaire de la population et des travailleurs contre les dangers des rayonnements ionisants». Cette directive en cours de transposition en droit français entérine les nouvelles grandeurs de protection - la dose équivalente et la dose efficace - et impose des limites de dose plus contraignantes pour les travailleurs exposés et pour le public.

Ces grandeurs, comme les précédentes (l'équivalent de dose à l'organe et l'équivalent de dose efficace, ICRP Publication 26, 1977) ne sont pas mesurables et l'ICRU (International Commission on Radiation Units and Measurements) a instauré depuis 1985 un système opérationnel basé sur le concept d'équivalent de dose en un point donné dans une sphère réceptrice «ICRU».

\footnotetext{
* Cet article a ćé présenté le mercredi 6 octobre 1999 dans le cadre de l'enseignement post-universitaire sur la « Dosimétrie opérationnelle en milieu médical, besoins et moyens » organisé conjointement par l'Institut national des sciences et lechniques nucléaires, la Société française de physique médicale et l'institut Gustave Roussy à Cadarache (INSTN),

** Institut national des sciences et techniques nucléaires, CEA/Cadarache, 13108 St-Paul-Lez-Durance. Mél didier.paul@cea.fr
} 
Les grandeurs opérationnelles définies par l'ICRU sont destinées à fournir des approximations raisonnables des grandeurs de protection de l'ICRP dans le but de respecter les limites réglementaires. Ces grandeurs sont donc des estimateurs «majorants $\left(H_{\mathrm{op}} \geq H_{\text {prot }}\right)$ développées pour les besoins et l'étalonnage pratique de l'instrumentation affectée aux surveillances de l'environnement et des personnes. La cohérence des grandeurs opérationnelles avec les grandeurs de protection a fait l'objet d'un groupe de travail ICRP/ICRU et d'une publication commune : la publication 74 de l'ICRP (1996) et le rapport 57 de l'ICRU portant sur les facteurs de conversion utilisés en protection radiologique (irradiation externe).

L'application des recommandations de l'ICRP et de l'ICRU nécessite une bonne compréhension des nombreux concepts, grandeurs et techniques d'étalonnage. Les décrets $n^{\circ}$ 98-1185 et 98-1186 du 24 décembre 1998 relatifs à la protection des travailleurs contre les dangers des rayonnements ionisants rendent obligatoire à partir du 31 décembre 1999 la dosimétrie opérationnelle pour toute opération se déroulant en zone contrôlée ou spécialement réglementée. Les modalités d'application sont fixées par l'arrêté du 23 mars 1999 et portent en particulier sur les caractéristiques requises en dosimétrie active (opérationnelle) et les conditions d'étalonnage.

Depuis 1985, les commissions internationales recommandent que les instruments de radioprotection soient étalonnés en terme de grandeurs opérationnelles.

\section{Définitions - Unités}

\subsection{Grandeurs de protection de l'ICRP}

Ce paragraphe se réfère aux recommandations de l'ICRP introduisant les concepts de "dose équivalente à l'organe» et de «dose efficace», grandeurs relatives au corps humain (ICRP Publication 60, 1991). Ces grandeurs remplacent la dose équivalente à l'organe ou au tissu et l'équivalent de dose efficace (ICRP Publication 26, 1977). Les changements sont rappelés dans le tableau I et portent principalement sur la définition des organes critiques considérés et l'utilisation de nouveaux facteurs de pondération radiologiques pour passer de la dose absorbée à la dose efficace. Les définitions des anciennes et nouvelles grandeurs de l'ICRP sont rappelées dans le tableau I.

Contrairement à l'équivalent de dose, fonction définie en un point et faisant intervenir le facteur de qualité utilisé par l'ICRP avant ses nouvelles recommandations, le facteur de pondération radiologique $W_{R}$ (ICRP Publication 60, 1991), déterminé par les caractéristiques du rayonnement incident sur l'organe, est appliqué à la dose absorbée moyenne $D_{T, R}$ dans l'organe considéré. Le nombre $T$ d'organes et les valeurs de $W_{T}$ (facteur de pondération tissulaire de l'organe $T$ ) sont donnés dans l'annexe A de la publication 60 de l'ICRP (1991). Il est important de signaler que ces grandeurs sont non mesurables et donc uniquement déterminées par calcul dans 


\section{TABLEAU I}

Recommandations de l'ICRP en 1977 (publication 26) et 1990 (publication 60). ICRP recommandations in 1977 (publication 26) and in 1990 (publication 60).

\begin{tabular}{|c|c|c|}
\hline Recommandations de 1977 & Recommandations de 1990 & Unités \\
\hline $\begin{array}{c}\text { Dose absorbée moyenne } \\
\text { sur un organe } D_{T}\end{array}$ & $\begin{array}{c}\text { Dose absorbée moyenne } \\
\text { sur un organe } D_{T}\end{array}$ & gray (Gy) \\
\hline $\begin{array}{c}\text { Facteur de qualité moyen } Q \\
\text { (calculé par interpolation à partir } \\
\text { d'un couple de valeurs numériques } \\
\text { de } Q \text { et de } T L E \text { ) }\end{array}$ & $\begin{array}{c}\text { Facteur de qualité selon une } \\
\text { nouvelle relation } Q=f(T L E) \\
\text { (formule mathématique) }\end{array}$ & $\begin{array}{c}\text { nombre sans } \\
\text { dimension }\end{array}$ \\
\hline $\begin{array}{l}\text { Facteur de qualité moyen } Q \\
\text { (Moyenne de } Q \text { pondérée } \\
\text { par le } T L E \text { sur le spectre } T L E \text { ) }\end{array}$ & $\begin{array}{l}\text { Facteur de pondération relatif } \\
\text { aux rayonnements } W_{R} \\
\text { (tiré des EBR sur les organes) }\end{array}$ & $\begin{array}{l}\text { nombre sans } \\
\text { dimension }\end{array}$ \\
\hline $\begin{array}{c}\text { Équivalent de dose } \\
\text { en un point : } \\
H=D \cdot Q\end{array}$ & $\begin{array}{c}\text { Équivalent de dose } \\
\text { en un point : } \\
H=D . Q \\
\text { (définition inchangée mais } \\
\text { utilisation limitée aux grandeurs } \\
\text { opérationnelles de I'ICRU) }\end{array}$ & sievert $(\mathbf{S v})$ \\
\hline $\begin{array}{c}\text { Equivalent de dose moyen } \\
\text { sur l'organe } T \text { : } \\
H_{T}=\text { Moyenne de } H \\
\text { sur l'organe. }\end{array}$ & $\begin{array}{l}\text { Dose équivalente } \\
\text { à l'organe T: } \\
H_{T}=W_{R} \cdot D_{T}\end{array}$ & sievert (Sv) \\
\hline $\begin{array}{l}\text { Équivalent de dose efficace : } \\
\qquad H_{E}=\sum_{T} w_{R} H_{T}\end{array}$ & $\begin{array}{l}\text { Dose efficace : } \\
E=\sum_{T} w_{R} H_{T}\end{array}$ & \\
\hline $\begin{array}{c}W_{T}=\text { Facteurs de pondération } \\
\text { relatifs aux tissus } \\
\text { (6 facteurs spécifiés) }\end{array}$ & $\begin{array}{c}W_{T}=\text { Facteurs de pondération } \\
\text { relatifs aux tissus } \\
(12 \text { facteurs spécifiés })\end{array}$ & sievert $(\mathrm{Sv})$ \\
\hline
\end{tabular}

des fantômes anthropomorphes. Par ailleurs, l'ICRP dans sa nouvelle publication, impose une nouvelle relation entre le facteur de qualité $Q(L)$ et le TEL ou $L$ (Transfert linéique d'énergie) bien qu'ayant abandonné le concept d'équivalent de dose en un point. Ce changement de valeurs se répercute sur les grandeurs opérationnelles de l'ICRU de type «équivalent de dose».

L'unité de dose absorbée est le «gray» (Gy), l'unité commune aux grandeurs de protection et grandeurs opérationnelles est le «sievert» (Sv).

\subsection{Grandeurs opérationnelles de l'ICRU pour la surveillance de l'environnement et de l'individu (d'après le paragraphe A.14 de la publication 60 de l'ICRP et le rapport 51 de l'ICRU)}

Les grandeurs ICRU données dans les rapports 39 et 51 de l'ICRU $(1985,1993)$ sont destinées à fournir des approximations raisonnables de la dose efficace et de la dose équivalente pour la peau (quand ces grandeurs sont calculées avec la relation 
$Q-L$ du tableau A-1 de la publication 60 de l'ICRP, 1991) et à permettre le respect de la réglementation. La détermination de grandeurs de remplacement liées à l'équivalent de dose efficace et à l'équivalent de dose à la peau (ICRP Publication 26, 1977 ; ICRU Rapport 39, 1985) a été faite séparément pour la surveillance de l'environnement (y compris les zones de travail) et la surveillance individuelle. Certaines conventions ont été utilisées pour cette surveillance. Toutes ces grandeurs sont basées sur le concept de l'équivalent de dose en un point donné dans la sphère ICRU composée d'oxygène ( $76,2 \%)$, de carbone ( $11,1 \%)$, d'hydrogène $(10,1 \%)$ et d'azote $(2,6 \%)$, de diamètre $=30 \mathrm{~cm}$ et de masse volumique $=1 \mathrm{~g} \mathrm{~cm}^{-3}$.

L'ICRU a modélisé le récepteur mais aussi l'émetteur en définissant des champs de rayonnement dérivés du champ de rayonnement réel pour la détermination des grandeurs opérationnelles. Les termes «expansé» et «unidirectionnel»sont donnés dans le rapport 39 de l'ICRU pour caractériser ces champs de rayonnement dérivés. Dans le champ expansé, la fluence et ses distributions angulaire et énergétique ont les mêmes valeurs dans le volume concerné que le champ réel au point de référence. Dans le champ expansé et unidirectionnel, la fluence et sa distribution en énergie sont les mêmes que dans le champ expansé, mais la fluence est unidirectionnelle. Le champ expansé est ainsi appliqué sur l'ensemble du dosimètre mais aussi sur tout le volume d'intérêt : le récepteur représenté par la sphère ICRU (Fig. 1).

\subsubsection{Surveillance de l'environnement}

Deux concepts liant le champ de rayonnement externe à la dose efficace et à la dose équivalente dans la peau sont introduits en vue de la surveillance de l'environnement et de la surveillance de zone. Le premier de ces concepts, l'équivalent de dose ambiant, $H^{*}(d)$, est approprié aux rayonnements fortement pénétrants, et le second, l'équivalent de dose directionnel, $H^{\prime}(d, \Omega)$ se réfère aux rayonnements faiblement pénétrants. Le caractère "pénétrant» des rayonnements dépend (selon la définition donnée dans le rapport 5 I de l'ICRU, 1993) du rapport à la valeur limite de l'équivalent de dose. Un rayonnement sera faiblement pénétrant si l'équivalent de dose le plus proche de la limite est l'équivalent de dose au niveau de la lentille de l'œil ou celui défini pour la peau, pour un rayonnement fortement pénétrant, l'équivalent de dose «corps entier» pour la dose efficace est le plus proche de la limite.

L'équivalent de dose ambiant, $H^{*}(d)$, en un point du champ de rayonnement, est l'équivalent de dose qui serait produit par le champ expansé et unidirectionnel correspondant, dans la sphère ICRU, à une profondeur « $d$ », sur le rayon opposé à la direction du champ unidirectionnel ; $d=10 \mathrm{~mm}$ pour les rayonnements fortement pénétrants, $0,07 \mathrm{~mm}$ pour les rayonnements faiblement pénétrants (dose à la peau) et $3 \mathrm{~mm}$ pour l'œil (Fig. 1). 
Détermination de $H^{*}(\mathrm{~d})$, champ expansé et unidirectionel

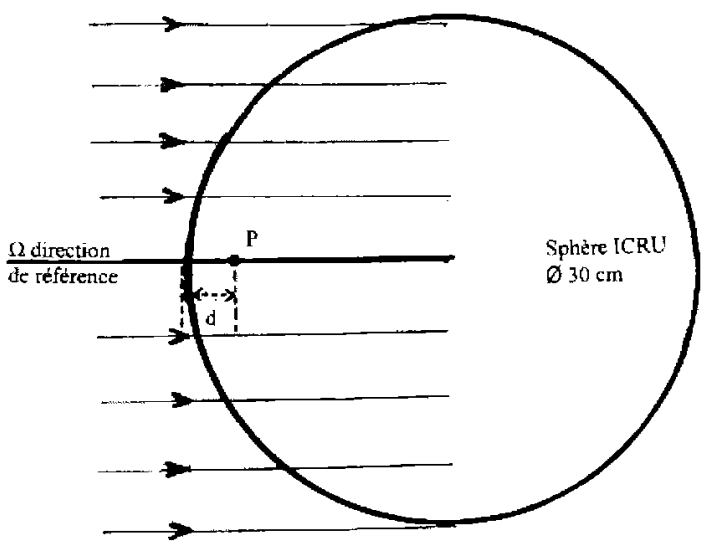

Détermination de $H^{\prime}(\mathrm{d}, \Omega)$, champ expansé

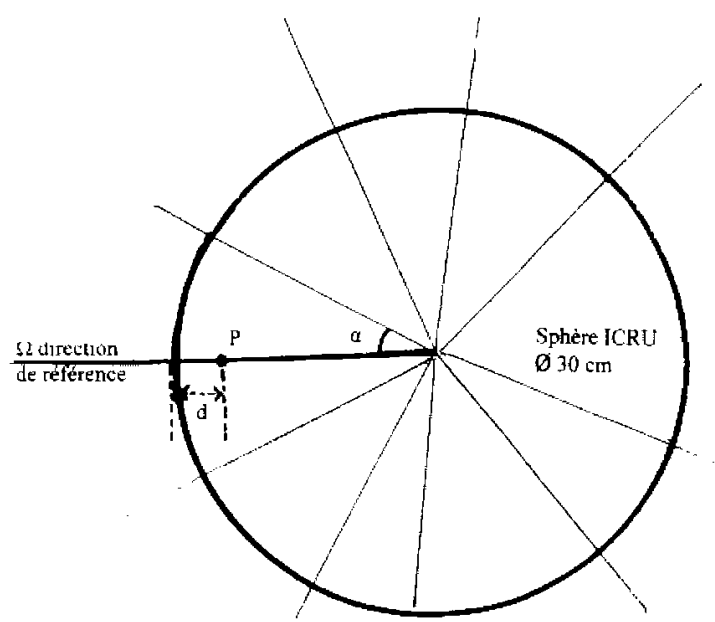

Fig. 1. - Détermination des grandeurs opérationnelles pour la surveillance des zones de travail et de l'environnement dans un champ dérivé (expansé) dont la fluence et ses distributions angulaire et énergétique ont dans la sphère ICRU les mêmes valeurs qu'au point de référence dans le champ réel (d'après Portal, 1989).

Determination of operational quantities for area monitoring in an expanded field of which the fluence and its directional and energy distributions have the same values throughout the ICRU sphere as in the actual field at the point of reference (from Portal, 1989). 
L'équivalent de dose directionnel, $H^{\prime}(d, \Omega)$ en un point du champ de rayonnement, est l'équivalent de dose qui serait produit par le champ expansé correspondant dans la sphère de l'ICRU, à une profondeur « $d$ », sur un rayon dont la direction $\Omega$ est spécifiée; $d=0,07 \mathrm{~mm}$ ou $3 \mathrm{~mm}$ (Fig. 1).

\subsubsection{Surveillance individuelle}

Au lieu de deux grandeurs définies dans le rapport 39 de l'ICRU (ICRU, 1985) pour la surveillance individuelle, un concept simplifié appelé équivalent de dose individuel ou personnel $H_{p}(d)$ est recommandé pour des rayonnements fortement et faiblement pénétrants. Selon L'ICRU (ICRU Rapport 51, 1993), $H_{p}(d)$ est l'équivalent de dose dans le tissu mou, à une profondeur appropriée « $d »$, sous un point spécifié du corps ; $d=10 \mathrm{~mm}, 3 \mathrm{~mm}$ ou $0,07 \mathrm{~mm}$.

Ces grandeurs sont dans leur principe adaptées aux besoins (paragraphe suivant) de la surveillance de zone et de la surveillance individuelle, rigoureusement définies, mesurables et traçables métrologiquement.

\section{Passage de la mesure à la dose absorbée (dosimétrie absolue et relative), à l'équivalent de dose et à la dose efficace}

La dosimétrie absolue relève plus de la spécialité du métrologiste que celle du spécialiste en radioprotection qui travaille avec des dosimètres dits relatifs. Cependant, la radioprotection, par déduction, s'appuie sur la dosimétrie absolue qui caractérise les références avec lesquelles sont étalonnés les instruments relatifs.

L'opération absolue d'un dosimètre consiste à atteindre la dose absorbée en un point d'un milieu $M$ au moyen d'un matériau détecteur $M$, de masse $m$, placé dans le milieu $M$ au point considéré.

La dose absorbée $\bar{D}_{M}$, dans le milieu matériau $M$ 'est par définition reliée à l'énergie moyenne $E$ déposée dans la masse $m$, proportionnelle à la valeur $g$ d'une grandeur mesurable. La relation fondamentale est la suivante :

$$
\bar{D}_{M^{\prime}}=\frac{\bar{E}}{m}=\frac{\omega g}{m} \quad \text { (d'après Blanc, 1990). }
$$

La dose $D_{M}$ recherchée est déduite de la mesure dans le matériau détecteur $M$ selon la théorie de la cavité, en remplaçant au point considéré, le matériau $M$ dú détecteur par le milieu considéré.

Trois dosimètres absolus répondent directement à la définition de la dose absorbée: le calorimètre, le dosimètre chimique et la chambre d'ionisation. 
La quantité caractéristique de ces dosimètres constitue le facteur de proportionnalité, $\omega$, entre la grandeur mesurée et l'énergie moyenne $\bar{E}$.

Les dosimètres relatifs ont une réponse qui ne peut pas être directement liée à l'énergie déposée dans le matériau détecteur, car de nombreux paramètres physiques, chimiques et géométriques interviennent dans la réponse. Les caractéristiques de ces dosimètres doivent être déterminées au moyen des dosimètres absolus.

En radioprotection, dosimétrie externe, la grandeur mesurable considérée est l'équivalent de dose, produit de la dose absorbée par le facteur de qualité du rayonnement $(H=D \cdot Q)$. Le passage de la mesure à l'équivalent de dose ou plutôt les équivalents de dose, définis dans l'ICRU, peut se faire selon plusieurs approches définies dans le rapport 47 de l'ICRU (ICRU, 1992) :

- une approche microdosimétrique (absolue) basée sur la définition même de l'équivalent de dose à partir de la mesure de l'énergie linéaire absorbée, grandeur proche du $T E L$ et du facteur de qualité $Q(y)$;

- une approche qui consiste à mesurer une autre quantité avec un dosimètre relatif, par exemple l'exposition ou le kerma pour les photons et à appliquer un coefficient de conversion calculé. Ces facteurs dépendant de l'énergie du rayonnement incident, une information sur le spectre en énergie est nécessaire. Cette méthode est couramment employée pour les étalonnages.

Dans le cadre habituel de la radioprotection, la détermination de l'équivalent de dose est généralement faite selon la deuxième approche. La réglementation imposant le respect des limites est basée sur le concept de la dose efficace, grandeur relative au corps humain. Grandeurs de protection et grandeurs opérationnelles peuvent être reliées à des grandeurs radiométriques comme la fluence de particules ou dosimétriques comme le kerma, la dose absorbée ou encore entre elles par le biais des coefficients de conversion, comme le montre la figure 2.

Les grandeurs opérationnelles actuellement utilisées pour l'étalonnage des dosimètres ont été à l'origine définies en 1985 pour être compatibles avec les grandeurs de la publication 26 de l'ICRP (ICRP, 1977). Depuis, l'ICRU a publié de nouveaux calculs concernant ses grandeurs en introduisant des données physiques récentes avec la révision de la relation Q-L de la publication 60 de l'ICRP (ICRU Rapport 47, 1992) pour les photons et les électrons. De nouveaux pouvoirs de ralentissement pour les protons et les particules alpha (ICRU Rapport 51, 1993) ont conduit à une révision des coefficients de conversion pour les neutrons (ICRP Publication 74, 1996).

La compatibilité des grandeurs opérationnelles $\left(H_{\mathrm{op}}\right)$ avec les nouvelles grandeurs de protection de la publication 60 de l'ICRP (ICRP, 1991) $\left(H_{\text {prot }}\right)$ suivant le principe général :

$$
H_{\text {prot }} \leq H_{\text {op }}
$$




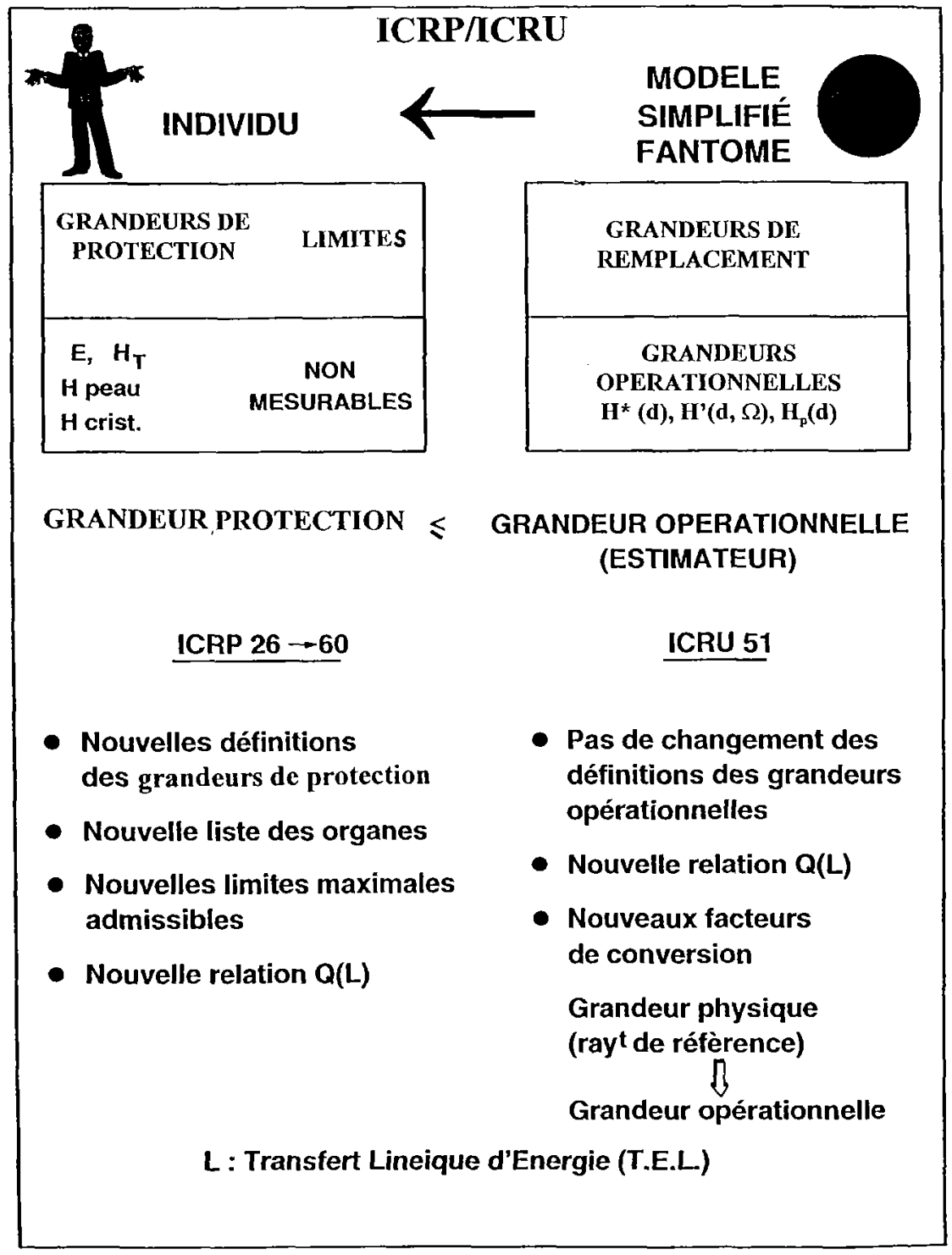

Fig. 2. - Estimation des grandeurs de protection de l'ICRP par les grandeurs opérationnelles de l'ICRU (d'après Chartier, 1998).

Estimation of ICRP protection quantities by the ICRU operational quantities (from Chartier, 1998). 
a été étudié par un groupe de travail ICRP/ICRU. Les figures 3 et 4 issues de la publication 74 de l'ICRP (ICRP, 1996) montrent quelques exemples de calculs de conversion entre les jeux de grandeurs pour les photons (Fig. 3) et les électrons (Fig. 4).

Le groupe de travail indique dans sa conclusion que dans la plupart des situations réellement rencontrées, les grandeurs opérationnelles définies par l'ICRU en 1985 constituent toujours une base satisfaisante pour les mesures de protection radiologique relativement à une irradiation externe en photon ou électron, ceci malgré tous les changements dans les deux jeux de grandeurs. La surestimation est typiquement de $20 \%$ ou plus, quelle que soit la géométrie d'irradiation.

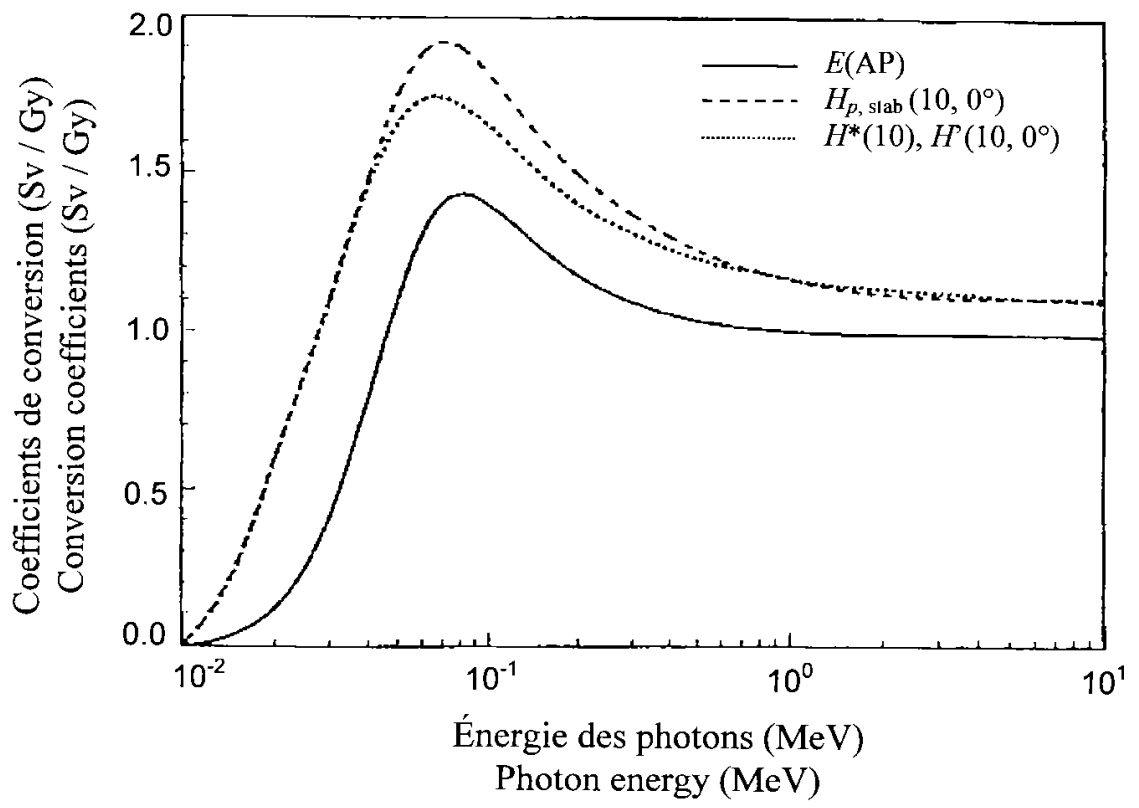

Fig. 3. - Coefficients de conversion du kerma dans l'air en grandeurs opérationnelles et en dose efficace en fonction de l'énergie des photons (géométrie d'irradiation AP) d'après la publication 74 de l'ICRP (1996).

Conversion coefficients of air kerma to operational quantities and effective dose as a function of photon energy (in AP irradiation geometry) from ICRP Publication 74 (1996). 
D. PAUL

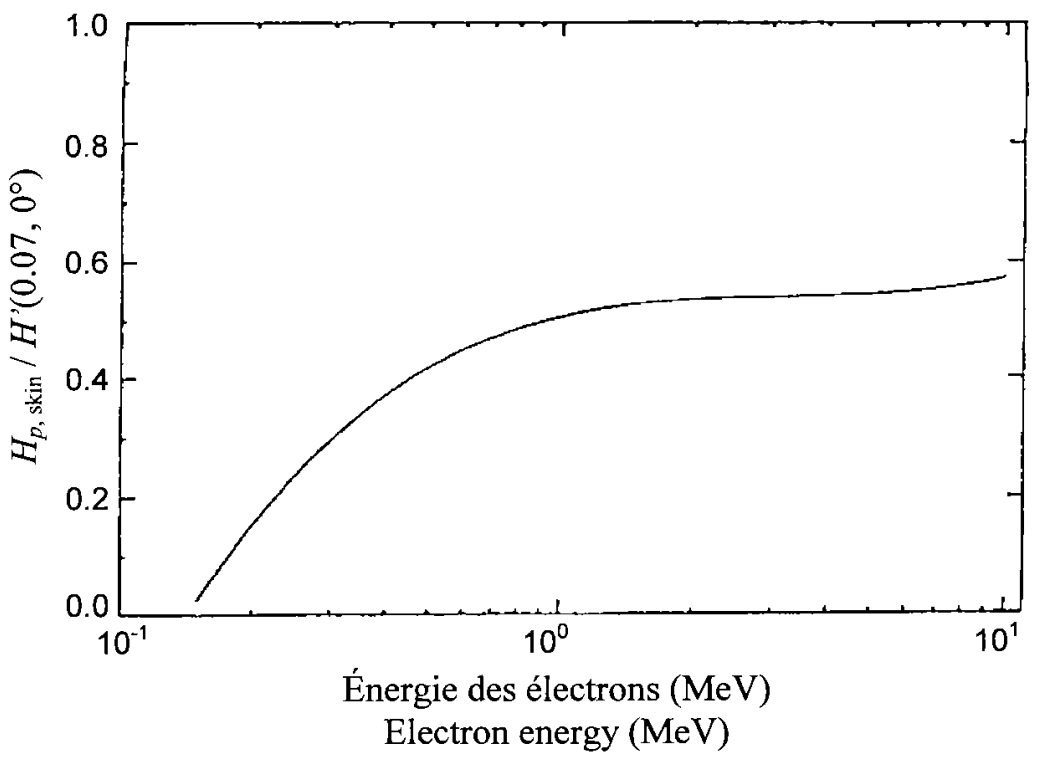

Fig. 4. - Rapport entre la dose équivalente à la peau et l'équivalent de dose directionnel $\left(H^{\prime}\left(0,07,0^{\circ}\right)\right.$ $\left.\approx H_{p}(0,07)\right)$ en fonction de l'énergie des électrons, d'après la publication 74 de l'ICRP (1996). The ratio of the skin dose, $H_{p, \text { skin }}$ to the directional dose equivalent $\left(H^{\prime}\left(0.07,0^{\circ}\right) \approx H p(0.07)\right)$ as a function of electron energy, from ICRP Publication 74 (1996).

\section{Caractéristiques des instruments de radioprotection requises en dosimétrie}

La qualité d'un instrument de radioprotection réside dans sa capacité à répondre correctement aux grandeurs opérationnelles $H^{*}(d)$ et $H^{\prime}(d, \Omega)$ définies pour les instruments de mesure d'ambiance et $H_{p}(d)$ pour les dosimètres individuels.

Dans le tableau II, pour les 2 catégories d'instruments et les différents rayonnements utilisés, pénétrants ou peu pénétrants, sont indiquées la quantité dosimétrique mesurée, fonction de l'étalonnage, et la grandeur opérationnelle considérée, issue de la conversion par un facteur approprié. Pour chacune de ces grandeurs, les réponses angulaire et énergétique de l'instrument sont rigoureusement étudiées.

Rappelons que la réponse d'un instrument est l'indication de la valeur de la quantité mesurée. 
GRANDEURS DE PROTECTION EN DOSIMÉTRIE EXTERNE

TABLEAU II

Données sur les fonctions de conversion (d'après le rapport 43 de l'ICRU, 1988).

Data on conversion functions (from ICRU report 43, 1988).

\begin{tabular}{|c|c|c|c|c|c|}
\hline Instrument & $\begin{array}{l}\text { Pouvoir du } \\
\text { rayonnement }\end{array}$ & $\begin{array}{l}\text { Équivalent } \\
\text { de dose }\end{array}$ & $\begin{array}{l}\text { Réponse } \\
\text { angulaire }\end{array}$ & $\begin{array}{c}\text { Type de } \\
\text { rayonnement }\end{array}$ & $\begin{array}{l}\text { Grandeur } \\
\text { primaire }\end{array}$ \\
\hline \multirow[t]{2}{*}{$\begin{array}{c}\text { Mesure } \\
\text { d'ambiance } \\
\text { (hors fantôme) }\end{array}$} & Pénétrant & $\begin{array}{c}H^{*}(10) \\
\text { (ambiant) }\end{array}$ & Isotrope & $\begin{array}{l}\text { Photon } \\
\text { Electron } \\
\text { Neutron }\end{array}$ & $\begin{array}{l}\text { Kerma dans l'air } \\
\text { Fluence } \\
\text { Fluence }\end{array}$ \\
\hline & Peu pénétrant & $\begin{array}{c}H^{\prime}(0,07) \\
\text { (directionnel) }\end{array}$ & Isodirectionnelle & $\begin{array}{l}\text { Photon } \\
\text { Electron } \\
\text { Neutron } \\
\text { Bêta }\end{array}$ & $\begin{array}{l}\text { Kerma dans l'air } \\
\text { Fluence } \\
\text { Fluence } \\
\text { Dose dans l'air } \\
\text { ou le tissu, ou } \\
\text { activité de la source }\end{array}$ \\
\hline \multirow[t]{2}{*}{$\begin{array}{l}\text { Dosimètre } \\
\text { individuel }\end{array}$} & Pénétrant & $\begin{array}{c}H p(10) \\
\text { (individuel) }\end{array}$ & Isodirectionnelle & $\begin{array}{l}\text { Photon } \\
\text { Electron } \\
\text { Neutron }\end{array}$ & $\begin{array}{l}\text { Kerma dans l'air } \\
\text { Fluence } \\
\text { Fluence }\end{array}$ \\
\hline & Peu pénétrant & $\begin{array}{c}H p(0,07) \text { et } \\
H p(3)\end{array}$ & Isodirectionnelle & $\begin{array}{l}\text { Photon } \\
\text { Electron } \\
\text { Neutron } \\
\text { Bêta }\end{array}$ & $\begin{array}{l}\text { Kerma dans l'air } \\
\text { Fluence } \\
\text { Fluence } \\
\text { Dose dans l'air } \\
\text { ou le tissu, ou } \\
\text { activité de la source }\end{array}$ \\
\hline
\end{tabular}

Dans le tableau II, compte tenu de la définition des différents équivalents de dose, la réponse angulaire de l'instrument doit être isotrope pour $H^{*}(10)$ c'est-à-dire que sa lecture doit être indépendante de la direction du rayonnement incident, non isotrope pour les autres équivalents de dose ou encore isodirectionnelle si la lecture varie avec la direction du rayonnement incident suivant la quantité opérationnelle considérée.

En dosimétrie individuelle, l'IEC (International Electrotechnical Commission) recommande pour les photons que le rapport entre une lecture à un angle $\alpha$ et une lecture $\alpha=0^{\circ}$ pour des angles allant de $+75^{\circ}$ à $-75^{\circ}$ soit au plus de $\pm 20 \%$ à l'énergie du ${ }^{137} \mathrm{Cs}(662 \mathrm{keV})$ et de $\pm 50 \%$ à l'énergie de $\mathrm{l}^{241} \mathrm{Am}(60 \mathrm{keV})$.

En ce qui concerne la réponse en énergie, le tableau III donne les normes fixant les spécifications relatives aux dosimètres individuels passifs et actifs (d'après Rannou et al., 1998).

Plusieurs dosimètres mesurent l'équivalent de dose dans les tissus adjacents au port du badge à $0,07,3$ et $10 \mathrm{~mm}$ de profondeur. Les dosimètres thermoluminescents qui sont de bons «équivalents-tissu» pour les photons ont des réponses angulaires et en énergie proches de l'idéal grâce à l'adjonction de filtres. Leur volume sensible est cependant encore trop épais pour la dosimétrie bêta. Malgré quelques détecteurs spécifiques (ultra-minces ou dopés au carbone), leur utilisation en 


\section{TABLEAU III}

Normes internationales fixant les spécifications relatives aux dosimètres individuels (d'après Rannou et al., 1998 et références incluses).

International standards specifying the requirements of personal dosemeters (from Rannou et al., 1998 and included references).

\begin{tabular}{|c|c|c|c|}
\hline Dosimètres & $\begin{array}{l}\text { Photographiques } \\
(20 \mathrm{keV}-9 \mathrm{MeV} \text { ) }\end{array}$ & $\begin{array}{l}\text { Thermoluminecents } \\
\text { dosimétrie individuelle }\end{array}$ & Électroniques \\
\hline Norme & ISO 1757 & CEI-ISO 1066 & $\begin{array}{l}\text { CEI } 1283(X, \gamma, \beta) \\
\text { CEI } 1323(\mathrm{n}), 1995 \\
\text { CEI } 1525(\mathrm{X}, \gamma, \beta \text { et } \mathrm{n}), 1997\end{array}$ \\
\hline $\begin{array}{l}\text { Réponse au } \\
\text { débit de dose }\end{array}$ & & & $\begin{array}{l}<20 \% \text { jusqu'à } 1 \mathrm{~Sv} \mathrm{~h}^{-1} \\
\left(^{137} \mathrm{Cs} \text { pour les photons, }\right. \\
{ }^{241} \mathrm{Am}-\mathrm{Be} \text { ou } \\
\text { les neutrons) }\end{array}$ \\
\hline $\begin{array}{l}\text { Réponse } \\
\text { en énergie }\end{array}$ & $\begin{array}{l} \pm 35 \% \text { pour les } \\
\text { photons entre } 20 \mathrm{keV} \\
\text { et } 1,2 \mathrm{MeV}\end{array}$ & $\begin{array}{l} \pm 30 \% \text { entre } 15 \mathrm{keV} \\
\text { et } 3 \mathrm{MeV} \text { pour les photons } \\
\pm 30 \% \text { entre } 50 \mathrm{keV} \\
\text { et } 3 \mathrm{MeV} \text { pour les } \\
\text { rayonnements } \beta\end{array}$ & $\begin{array}{l} \pm 30 \% \text { entre } 50 \mathrm{keV} \\
\text { et } 1,5 \mathrm{MeV} \text { par rapport } \\
\text { au } 137 \mathrm{Cs} \text { pour les photons, } \\
-50 \%,+100 \% \text { pour } 6 \mathrm{MeV} \\
\text { (test uniquement sur } \\
\text { site réacteur) } \\
\pm 50 \% \text { pour les neutrons } \\
\text { entre } 100 \mathrm{keV} \text { et } 15 \mathrm{MeV}\end{array}$ \\
\hline $\begin{array}{l}\text { Réponse } \\
\text { angulaire }\end{array}$ & $\begin{array}{l} \pm 30 \% \text { pour angles } 0^{\circ} \\
\pm 30^{\circ}, \pm 45^{\circ}, \pm 60^{\circ} \text { et } \\
\text { aux énergies } 58 \mathrm{keV} \text { ou } \\
{ }^{241} \mathrm{Am} \text { et } \gamma \text { du }{ }^{137} \mathrm{Cs} \\
\text { ou }{ }^{60} \mathrm{Co}\end{array}$ & $\begin{array}{l}15 \% \text { pour les angles } 0^{\circ} \text {, } \\
\pm 20^{\circ}, \pm 40^{\circ}, \pm 60^{\circ} \text { et pour } \\
\text { les photons de } 60 \pm 5 \mathrm{keV}\end{array}$ & $\begin{array}{l}\text { Entre } 0^{\circ} \text { et } \pm 75^{\circ}: \\
\pm 20 \% \text { pour }{ }^{137} \mathrm{Cs} \\
\pm 50 \% \text { pour }{ }^{241} \mathrm{Am} \\
\pm 30 \% \text { pour }{ }^{241} \mathrm{Am}-\mathrm{Be} \text { ou }{ }^{252} \mathrm{Cf}\end{array}$ \\
\hline
\end{tabular}

dosimétrie bêta est réservée aux énergies élevées (par exemple, ${ }^{90} \mathrm{Sr}-{ }^{90} \mathrm{Y}$ ). Les films photographiques avec filtres sont toujours utilisés bien qu'étant de mauvais «équivalents-tissu ». L'indépendance énergétique pour différents angles d'incidence est en général difficile à assurer surtout aux basses énergies pour les photons.

En dosimétrie de zone pour les photons, les instruments de mesure d'ambiance qui posent des problèmes de sensibilité excessive au-dessous de $100 \mathrm{keV}$ en terme d'exposition ou de kerma varient moins fortement en fonction de l'énergie pour la mesure de $H^{*}(10)$. Cette conversion heureuse en équivalent de dose permet à beaucoup d'instruments mesurant l'exposition ou le kerma de déterminer $H^{*}(10)$ par un simple changement d'échelle pour passer des anciennes grandeurs et unités aux nouvelles.

Pour le rayonnement bêta, l'instrument le plus utilisé en métrologie est la chambre à extrapolation qui présente une réponse quasi isodirectionnelle relativement à $H^{\prime}(0,07, \Omega)$.

La dosimétrie des neutrons constitue un chapitre à part entière qui dépasse le cadre de cet exposé. 
La dosimétrie opérationnelle est généralement associée à la dosimétrie active, comme décrite dans l'arrêté du 23 mars 1999, au service des principes d'optimisation et de limitation en radioprotection. Les dosimètres électroniques (diodes silicium) règnent en maître pour les photons. Malgré ses petites dimensions, le détecteur présente une bonne sensibilité. Par contre le silicium n'est pas un bon «équivalent-tissu » compte tenu de son numéro atomique relativement faible. En dosimétrie individuelle, ils sont étalonnés en $H_{p}(d)$, leur réponse entre dans les limites de variation autorisées par la norme CEI ă partir de $60 \mathrm{keV}$ jusqu'à $3 \mathrm{MeV}$. Notons que certains dosimètres sont munis d'une diode supplémentaire pour permettre la mesure de $H_{p}(0,07)$ à partir d'une énergie de l'ordre de 15 à $20 \mathrm{keV}$.

\section{L'étalonnage : grandeurs de référence et procédures associées}

On entend par étalonnage d'un instrument, la détermination du facteur d'étalonnage $N$ par lequel on multiplie la lecture de l'instrument pour obtenir l'équivalent de dose désiré :

$$
N=H / M
$$

où $M$ est la lecture de l'instrument dans un champ de référence où l'équivalent de dose est connu.

Pour bien étalonner un instrument, il faut étudier sa réponse angulaire, spectrale et la manière dont elle dépend d'autres paramètres. L'ensemble des procédures à accomplir et les caractéristiques requises apparaissent dans les normes IEC et ISO qui définissent également les champs de référence appropriés pour réaliser ces étalonnages (photon, électron, neutron).

À l'heure actuelle, les laboratoires de métrologie utilisent encore les quantités primaires du tableau II : kerma dans l'air, fluence, dose absorbée dans l'air ou le tissu, activité de la source, comme grandeurs de référence et transforment ces données en grandeurs opérationnelles par l'intermédiaire de facteurs de conversion calculés, que l'on trouve dans le rapport 47 de l'ICRU et la publication 74 de l'ICRP (ICRU, 1992; ICRP, 1996).

La procédure pour les dosimètres d'ambiance bêta est différente de celle des photons et des neutrons puisqu'elle n'utilise pas de facteurs de conversion calculés. Les dosimètres sont étalonnés en terme de dose absorbée sous $7 \mathrm{mg} \mathrm{cm}^{-2}$ de matériau «équivalent-tissu». Cette mesure est équivalente à celle de $H^{\prime}(0,07)$ avec un facteur de qualité égal à 1 .

L'étalonnage des dosimètres individuels se fait en présence d'un fantôme adéquat qui reproduit le rayonnement diffusé par le corps de l'individu, contribution intégrée dans la définition de $H p(d)$. 
D. PAUL

Pour les photons, le facteur d'étalonnage de beaucoup d'instruments est relativement indépendant de l'énergie. La procédure d'étalonnage est complexe, plusieurs méthodes sont utilisées pour réduire l'incertitude sur le facteur d'étalonnage (normes ISO). Pour obtenir une précision de mesure de $30 \%$, il faut réaliser un étalonnage de l'instrument avec une précision inférieure à $10 \%$ ce qui implique des références connues à moins de $5 \%$.

Le rapport 47 de l'ICRU (ICRU, 1992) recommande le fantôme PMMA de $30 \mathrm{~cm}$ $\times 30 \mathrm{~cm} \times 15 \mathrm{~cm}$ dont la masse est proche de la sphère ICRU et dont les caractéristiques de rétrodiffusion sont proches de celles du corps humain.

\section{Conclusion}

Une nouvelle génération de dosimètres arrive au service de l'optimisation et du respect des nouvelles limites de la radioprotection; ils ont pour fonction d'assurer le passage de la mesure aux grandeurs opérationnelles. Pour les rayonnements photoniques fortement pénétrants, les mesures d'ambiance et sur l'individu sont déjà «opérationnelles» avec les instruments actuels de la dosimétrie active. Les dosimètres individuels comme les thermoluminescents et les films ont besoin d'être modifiés légèrement pour déterminer $H_{p}(10)$ et améliorer l'isodirectionnalité de leur réponse angulaire. Pour les rayonnements faiblement pénétrants et en mesure d'ambiance, il est difficile d'avoir à la fois un bon dosimètre pour les rayonnements bêta et gamma. Des détecteurs spéciaux pour le rayonnement bêta sous forme de sondes déportées apparaissent sur le marché. Au niveau des dosimètres individuels, de faibles épaisseurs en matériaux «équivalents-tissu» $(0,07 \mathrm{~mm})$ sont recherchées en amont de la zone sensible pour avoir une réponse angulaire et en énergie correcte (ICRU Rapport 47, 1992).

La réglementation actuelle (arrêté du 23 mars 1999) prend en compte l'ensemble des techniques de dosimétrie individuelle (passive et active) éprouvées suivant les concepts physiques de l'ICRU et les critères de l'IEC afin de répondre aux nouvelles exigences de la directive européenne et afin de respecter les limites de la dose efficace.

Remerciements : Je tiens à remercier J.L. Chartier (IPSN), membre du groupe de travail ICRP/ICRU pour son aide précieuse dans divers domaines et pour son action au sein du groupe de travail GTNI qu'il a présidé. Cet article issu d'un enseignement est « introductif» au rapport complet sur l'application des nouvelles grandeurs opérationnelles, préparé actuellement par le groupe GTN1 du CTHIR (IPSN), rapport qui est annoncé pour le courant de l'année 2000. 


\section{GRANDEURS DE PROTECTION EN DOSIMÉTRIE EXTERNE}

\section{RÉFÉRENCES}

Arrêté du 23 mars 1999 précisant les règles de la dosimétrie externe des travailleurs affectés à des travaux sous rayonnements en application des articles 20 bis et 25 -I du décret du 28 avril 1975 modifié et des articles 31 bis et 34-I du décret du 2 octobre 1986 modifié.

Blanc D. (1990) Les rayonnements ionisants, détection, spectrométrie, dosimétrie. Masson, Paris.

Chartier J.L. (1998) Résultats des travaux du groupe de travail ICRP/ICRU sur les conséquences des recommandations de l'ICRP Publication 60 en dosimétrie des neutrons, Journées SFRP/SPT sur la dosimétrie des neutrons, INSTN/Cadarache.

Décrets $n^{\circ}$ 98-1 185 et 98-1 186 du 24 décembre 1998 modifiant le décret n 86 - 1103 du 2 octobre 1986 modifié relatif à la protection des travailleurs contre les dangers des rayonnements ionisants.

Directive 96/29/Euratom du Conseil du 13 mai 1996, fixant les normes de base relatives à la protection sanitaire de la population et des travailleurs contre les dangers résultant des rayonnements ionisants.

ICRP Publication 26 (1977) Recommendations of the ICRP, Ann. ICRP 1 (3).

ICRP Publication 60 (1991) Recommendations of the ICRP, Ann. ICRP 21 (I-3).

ICRP Publication 74 (1996) Conversion Coefficients for use in Radiological Protection against External Radiation, Ann. ICRP 26 (3-4).

ICRU Rapport 39 (1985) Détermination des équivalents de dose dus aux sources externes de rayonnement, Pergamon Press, Oxford.

ICRU Rapport 43 (1988) Determination of Dose Equivalents from External Radiation Sources, Part 2, Pergamon Press, Oxford.

ICRU Rapport 47 (1992) Measurement of Dose Equivalents from External Photon and Electron Radiations. Pergamon Press, Oxford.

ICRU Rapport 51 (1993) Quantities and Units in Radiation Protection Dosimetry. Pergamon Press, Oxford. Portal G. (1989) Communication personnelle.

Rannou A, Barthe J, Aubert B, Bregeon B., Champlong J., Colson P., Espagnan M., Herbaut Y., Thévenin J.C., Valero M. (1998) État de l'art des techniques de dosimétrie individuelle et analyse des besoins, Radioprotection 33, $405-433$. 